\title{
Is SARS-CoV-2 present in the periodontium? A post-mortem study
}

\section{Mohammed Adam ${ }^{1}$}

\section{A commentary on}

Fernandes Matuck B, Dolhnikoff M, Maia G V A et al.

Periodontal tissues are targets for Sars-Cov-2: a post-mortem study.

J Oral Microbiol 2020; 13: 1848135.

\section{Abstract}

Aims To investigate the presence of SARS-CoV-2, the virus responsible for COVID-19 infection, in the periodontal tissues of deceased individuals with a positive SARS-CoV-2 nasopharyngeal swab test.

Sample selection Seven deceased individuals, who were admitted to the intensive care unit of the Hospital das Clínicas of the Medicine School of Sao Paulo University for invasive mechanical ventilation following a positive nasopharyngeal COVID-19 test result, underwent a post-mortem examination. Individuals with comorbidities were included. Exclusion criteria included a current smoking habit.

Design A cross-sectional study involving a videoscope-guided autopsy to obtain a periodontal tissue sample. The plastic body wrapping was incised in the facial region, allowing the insertion of a video endoscope. A single sample of periodontal tissue (junctional epithelium, adjacent oral epithelium and underlying connective tissue) was obtained from the mesial interproximal papilla of the maxillary first molar (or if absent, the mesial tooth). The sample was cleaned using an enzymatic detergent (Riozyme) to mimic the effects of salivary flow and was collected using blunt dissection. The sample was divided into two samples for either histopathological analysis (haematoxylin and eosin stain) or molecular analysis. Real-time reverse transcriptase polymerase chain reaction (rRT-PCR) was used to test for the presence of the envelope (E) gene of SARS-CoV-2. Data on comorbidities, smoking status, period of hospitalisation and time from onset of symptoms to death were also collected.

Data analysis Descriptive analysis was performed and results were presented as a cross tabulation. The presence of SARS-CoV- 2 in samples was presented as the rRT-PCR cycle threshold value and the histopathological analysis results were Boolean (that is, presence/ absence of vacuolisation or nuclear pleomorphism).

Results A total of seven individuals were examined: four women and three men. The age range was $8-74$ years and the range of periods between onset of symptoms and death was 10-31 days. SARS-CoV-2 was detected in five samples, with a mean cycle threshold of 31.38 (range 27.28-36.55). Nuclear pleomorphism and vacuolisation were found in two and three cases, respectively.

Conclusions SARS-CoV-2 genetic material was present in most periodontal tissue samples of individuals with COVID-19. The sample size, however, was small and only included severely ill individuals with prolonged periods of hospitalisation. Consequently, the applicability of the findings to the general population is limited.

\section{GRADE rating}
Practice point
- Clinicians should continue to practise universal infection control precautions.

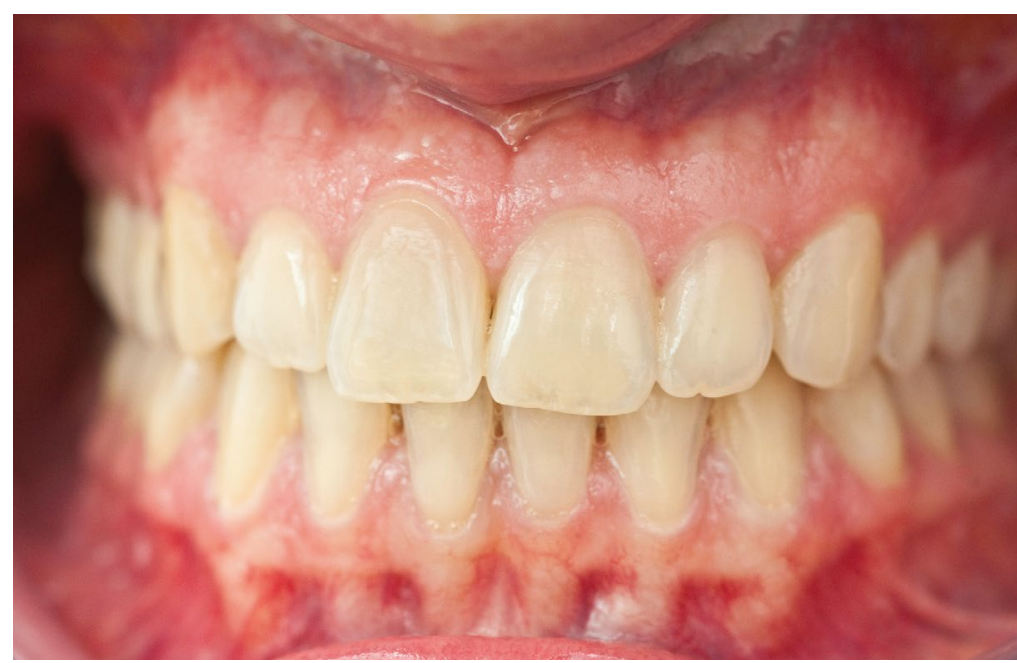

\section{Commentary}

The recent spike in research investigating potential relationships between the oral cavity and COVID-19 infection is understandable considering the well-recognised associations between oral and systemic health. It is widely accepted that periodontitis and certain systemic diseases are bidirectionally related. Suggested hypotheses include the periodontium acting as an entry point for pathogens leading to systemic bacteraemia, and as a reservoir of pro-inflammatory mediators and bacterial products which can spill into the systemic circulation. ${ }^{1}$ It is therefore reasonable to theorise that periodontal health and COVID-19 infection may be related.

This study aimed to investigate whether genetic material from SARS-CoV-2 was present in the periodontal tissues. The rationale behind this was that SARS-CoV-2 has been found in saliva and gingival crevicular fluid (GCF), and that periodontal tissue cells express angiotensin-converting enzyme 2 receptors via which the virus enters human cells. ${ }^{2,3}$ The authors also aimed to describe a novel minimally invasive approach to obtain a periodontal tissue sample during a post-mortem.

The results of the study are somewhat unsurprising given that detection of viral genetic material in the periodontium is not uncommon in human herpes virus infections. SARSCoV-2 has been shown to be present in saliva and GCF.,5 Purported mechanisms include the periodontium acting as an entry point for the virus or dissemination of the virus from the systemic circulation into the periodontal tissues. ${ }^{6}$ Since GCF, an inflammatory exudate, contains SARS-CoV-2, it would seem that 
the latter explanation is more plausible. It should be noted that rRT-PCR testing demonstrates the presence of viral ribonucleic acid (RNA) and not the presence of viable and contagious viral particles. It is known that in the upper respiratory tract, viral RNA shedding may occur for several weeks; however, such studies have demonstrated that high viral RNA load does not necessarily correlate with culturable and viable virus presence. ${ }^{7}$

While acknowledging the difficulties in organising rigorous, large and multicentre studies during a pandemic, this study presents clear limitations. The sample size was small and unrepresentative of most cases of COVID-19 which do not lead to hospitalisation. The sampling process is unclear, and while attempts were made to superficially decontaminate the sample, viral RNA from the GCF may have been inadvertently included in the sample. The design, although described as cross-sectional in nature, resembles a case series. This has been reflected in the GRADE rating. While this study demonstrates the presence of SARS-CoV-2 in the periodontium, its clinical relevance and generalisability to most cases of COVID-19 may be limited.

\section{Author affiliation}

${ }^{1}$ University of Liverpool, Liverpool, UK.

Correspondence to: Mohammed Adam

\section{References}

1. Lopez-Lago A, Choudhury M T, Sharma P. What are the links between periodontitis and systemic disease? Dent Update 2019; 46: 914-917.

2. To K K, Tsang O T, Yip C C et al. Consistent Detection of 2019 Novel Coronavirus in Saliva. Clin Infect Dis 2020; 71: 841-843.

3. Gabriele L, Morandini A C, Dionísio T, Santos C. Angiotensin II Type 1 Receptor (AT1) Knockdown Impairs Interleukin (IL)-1 $\beta$-Induced Cytokines in Human Periodontal Fibroblasts. J Periodontol 2016; 88: 1-15.

4. Kubar A, Saygun I, Ozdemir A, Yapar M, Slots J. Real-time polymerase chain reaction quantification of human cytomegalovirus and Epstein-Barr virus in periodontal pockets and the adjacent gingiva of periodontitis lesions. I Periodontal Res 2005; 40: 97-104.

5. Gupta S, Mohindra R, Chauhan P K et al. SARS CoV 2 Detection in Gingival Crevicular Fluid. J Dent Res 2021; 100: 187-193.

6. Badran Z, Gaudin A, Struillou X, Amador G, Soueidan A. Periodontal pockets: A potential reservoir for SARS CoV 2? Med Hypotheses 2020; 143: 109907.

7. Cevik M, Tate M, Lloyd O, Maraolo A E, Schafers J, Ho A. SARS CoV 2, SARS-CoV, and MERS-CoV viral load dynamics, duration of viral shedding, and infectiousness: a systematic review and meta-analysis. Lancet Microbe 2021; 2: 13-22.

Evidence-Based Dentistry (2021) 22, 60-61.

https://doi.org/ 10.1038/s41432-021-0184-0 